\title{
Síndrome de tensión premenstrual. Estudio sintomatológico de 302 casos.
}

\author{
Guillermo Acosta Osio MD**, Hernando Franco N. MD***
}

RESUMEN: Conocer de la importancia actual del Síntrome de Tensión Premenstrual (STP), y cómo afecta éste a la mujer en la vida laboral y afectiva, decidimos realizar un estudio inicial al azar con 317 estudiantes de carreras paramédicas de 1o. a 5o. año. Se incluyeron 302 casos,

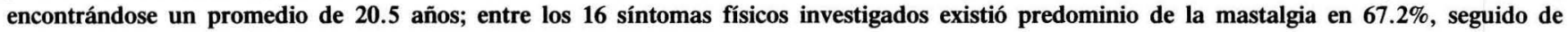
hinchazón abdominal $66.2 \%$, acné en el $61 \%$ y cefalea en el $50 \%$ de las pacientes.

Entre los 9 síntomas psicológicos analizados encontramos predominio de la irritabilidad en 58.2\%, seguido por la depresión en $52 \%$; y entre los 5 síntomas conductuales predominaron afectación laboral y escolar.

El 55\% de las pacientes utilizan drogas para calmar los síntomas.

También fue estudiado la fecha de inicio de los síntomas, el número de síntomas presentados, la duración de los mismos, su relación con la menarquia y la influencia de éste síndrome en la autoimagen femenina.

Entre las conclusiones tenemos que el $\mathbf{6 4 . 5 \%}$ de las pacientes cursan con STP leve, el $25.2 \%$ con STP moderado, y el $7.3 \%$ con STP severo. Menos del $1 \%$ de las mujeres consultan a médicos por éste padecimiento y el 55\% se automedican. No existe un tratamiento específico para este padecimiento pues su etiología aún es incierta.

PALABRAS CLAVES: Síndrome premestrual, Estudio epidemiológico, Síntomas físicos, Síntomas psicológicos.

SUMMARY: Knowing the recent importance of the Premenstrual Syndrome (PMS) and the way how it affects women's laboral and affective life, we decided to carry out a randomized study with 317 of paramedical careers.

302 cases were selected with a mean age of 20,5 years old.

We have examined 16 physical symptoms, the main of wich were considered to be mastalgia (67,2\%), abdominal bloating (66,2\%), acné (61\%) and headache $(50 \%)$.

Among the 9 analyzed psychological sympstoms the prevalent features were irritability $(58,2 \%)$, depression (52\%) and among the 5 analyzed, behavior related symptoms it stood out laboral and schoolar affectation. The $55 \%$ of the patients used pharmacs to relieve their symptoms.

It has been also studied the time of symptoms appearance, the kind of developed symptoms, their duration, their relation with menarche and the influence of PMS in the femenine self-image of the patients.

The results of this study show, that the $64.5 \%$ of the patients develops slight PMS, moderate in $25.2 \%$ and severe in $7.3 \%$ of the cases. Less than $1 \%$ of the women visit the gynecologists because of these complaints and $55 \%$ are selfmedicated.

It doesn't exist specific treatment for this syndrome since its etiology is yet uncertain.

KEY WORDS: Premenstrual Syndrome, Epidemiologycal Study, Physical Symptoms, Physchological Symptoms.

\section{Síndrome de tensión premenstrual}

Se entiende por Síndrome de Tensión Premenstrual (STP), a una serie de modificaciones de índole física, psicológica y de la conducta, que se presentan durante el período que precede a la menstruación y disminuyen o desaparecen al iniciarse el flujo menstrual (1). Este padecimiento es más común con vida sexual activa, intelectuales, residentes en zonas urbanas, longuilíneas y pertenecientes a clases socioeconómica acomodadas; incluso consideran algunos autores (2) que existen factores desencadenantes como son el aborto provocado, infecciones de genitales o después de un parto; pero por lo general la mayoría de estas mujeres presentan

\footnotetext{
* Presentado en el XIII Congreso Latinoamericano y X Congreso Uruguayo de Ginecología y Obstetricia.

** Profesor y Director Dpto. Ginecología y Obstetricia, Hospital Universitario Metropolitano.

*** Residente III año Ginecología y Obstetricia, Universidad Metropolitana y Hospital Universitario Metropolitano.
}

inestabilidad psicoafectiva y padecen de desequilibrios neurovegetativos y como consecuencia se presentan comúnmente riñas matrimoniales, deficiencia y ausentismo laboral así como aislamiento social $(3,4)$.

\section{Etiología}

Insuficiencia de progesterona:

Por mucho tiempo se consideró que esta era la causa del STP, debido a un exceso de estrógenos; inclusive Frank, (5) que fue el primero en describir el síndrome, propuso la venisección para disminuir la concentración de hormonas, de lo convencido que estaba era debido a un exceso de estrógenos. Pero hace poco algunos autores $(4,6)$ no encontraron diferencia en la concentración de progesterona durante la fase progestacional de testigos y de mujeres que padecían STP, incluso ni siquiera encontraron elevación de los estrógenos, sino que estos estaban normales y las concentraciones de progesterona eran relativamente altas. Lo que si es cierto es que no existe correlación entre las publicaciones, sino 
que más bien se plantean fuertes controversias respecto a considerar esta teória hormonal como base del STP. En estudios doble ciergo realizados por Reid y Yen (1), utilizando progestágenos y placebo como tratamiento, no encontraron que la homonoterapia sea mejor que los placebos, aunque se considera que dosis farmacológicas de progesterona pueden actuar de manera todavía desconocida por la cual se presenta el aparent alivio de los síntomas del STP (7) de todas formas Strickler (4) plantea la idea de una etiología hormonal ovárica basado en que además que las concentraciones periféricas de hormonas pueden no reflejar con exactitud las concentraciones en el sistema nervioso central, existen receptores para estrógenos y progesterona en el cerebro y que estos modifican la actividad eléctrica, enzimas y neurotrasmisores, lo cual puede exacerbar los síntomas depresivos y trastornos afectivos que se relacionan con el ciclo menstrual, además que el tratamiento con progesterona parece adecuado para tratar la agitación, ansiedad, insomnio, y otros de los síntomas del STP.

\section{Retención de líquidos}

Si bien se creía en la ídea que en el STP, existía retención generalizada de líquidos, ya que la mayoría de las mujeres refieren hinchazón de alguna parte del cuerpo, estudios realizados por Reid (8) en los que valora la ganancia de peso, el intercambio total de sodio y el agua total corporal no ha podido demostrar existencia de un patrón de retención de líquidos en estas mujeres; al parecer lo que existe es una desviación de los líquidos sin retención de agua y sodio. La progesterona actúa como un agonista parcial de la aldosterona que se encuentra aumentada en la fase progestacional pero sin diferencias en la concentración plasmática entre mujeres con STP y testigos; induciendo una natriuresis temporal seguida de una aumento compensador en el eje renina angiotensina aldosterona. El riñón puede metabolizar la progesterona hacia el mineralocorticoide desoxicorticosterona que se encuentra aumentado en la fase lutea, por lo tanto el efecto natriurético de la progesterona parece compensarse por cambios apropiados en hormonas que conservan el sodio corporal $(1,4,9)$

\section{Prolactina}

La prolactina, que tiene ritmo circadiano con un pico máximo durante el sueño, aumenta aproximadamente en la mitad del ciclo y durante la fase progestacional y, estimula las glándulas mamarias. Pero aunque ésta regula el equilibrio de líquidos y electrolitos en peces y aves, está demostrado que no tiene efectos en el ser humano(8). El tratamiento con agonistas dopaminérgicos tipo bromocritina, ha demostrado que alivia los síntomas mamarios sin efectos sobre otros síntomas del STP, por lo tanto las anormalidades de la prolactina ya no se postulan como causa de este síndrome $(1,4,10)$.

\section{Hipoglucemia}

La hipoglucemia ha sido postulada como causa del STP, basado en que algunos síntomas premenstruales semejan síntomas de hipoglicemia, pero cabe anotar que la hipoglicemia se presenta también en mujeres que no sufren del STP, y que éste muy rara vez se alivia con la ingesta de alimentos $(1,4)$.
Existen estudios (11) en los cuales se mide la respuesta de la glucosa plasmática, insulina y glucagón en diferentes fases del ciclo durante la prueba de tolerancia a la glucosa, sin observarse diferencias significativas entre mujeres normales y aquellas con hipoglucemia premenstrual, por lo tanto esta teoría ya no tiene validez.

\section{Deficiencia de piridoxina}

La Vitamina B6, que es cofactor en la síntesis de serotonina y dopamina a partir de aminiácidos y en la formación de prostaglandinas a partir de ácidos grasos esenciales, cuando se encuentra disminuida a nivel cerebral, presenta el paciente síntomas de ansiedad, depresión e irritabilidad como ha sido demostrado en las pacientes que ingieren anticonceptivos (4); pero la respuesta clínica al tratamiento con piridoxina ha sido muy variada, presentando algunas pacientes mejoría notoria, y otras en cambio no presentan ningún beneficio. Como muy pocos estudios han sido controlados con placebo, no se puden emitir conclusiones al respecto y el efecto beneficioso de la piridoxina sobre la irritabilidad y depresión se presentó tanto en la fase folícular como en la fase luteínica, no se observa ninguna relación específica entre la deficiencia de Vitamina B6 y el STP $(1,4,12)$.

\section{Endorfinas}

Se cree que la progesterona sola o combinada con los estrógenos pueden estimular la actividad central de los péptidos opiaceos endógenos; estos péptidos que actúan como neuromoduladores, con efectos sobre el humor, la secreción endocrina y la conducta, al encontrarse aumentados en la fase lutea media producen disminución en la liberación de neuroepinefrina o dopamina; por la inhibición de estos péptidos sobre el sistema de aminas biógenas, se presentan síntomas como depresión, cansancio, hambre, fatiga y constipación. Al disminuir la acción ejercida por los opiaceos a medida que se aproxima la menstruación, se produce un rebote de hiperactividad de estos neurotransmisores lo que puede dar lugar a irritabilidad, ansiedad, tensión, hostilidad, diarrea, etc. La severidad de los síntomas del STP, parece ser debida a si la exposición a los péptidos opiaceos endógenos es crónica o intensa o si la deprivación de los mismos es brusca (13).

Se ha demostrado también que estos péptidos tienen un efecto inhibitorio sobre las gonadotrofinas, ya que al administrar la naloxona-antagonista de los receptores opiáceos, durante la fase luteínica del ciclo cuando los niveles de gonadotropinas se encuentran normalmente disminuidos; se produce un aumento en la concentración sérica de $\mathrm{LH}$, mientras que en la fase folicular precoz, la administración de naloxona no produce ningún cambio en los niveles de $\mathrm{LH}$ lo cual sugiere que la inhibición de las gonadotrinas por los péptidos opiáceos endógenos es mínima en esta fase del ciclo (14).

Estudios realizados por Chuong y colaboradores (15) midiendo las concentraciones de $\mathrm{B}$ endorfinas en mujeres sanas y con STP encontraron una disminución significativa durante la fase progestacional en las mujeres que padecían STP, contra un aumento en las mujeres sanas, por lo tanto ellos manifiestan haber identificado un marcador bioquímico para el STP pero no han podido diferenciar con la medición de 
estas endorfinas periféricas, aquellas mujeres que tienen empeoramiento de su depresión de aquellas, en las cuales se les adjudica el diagnóstico erróneo de STP; además que la concentración de endorfinas en plasma no refleja la concentración de opiáceos en el sistema nervioso central por lo tanto en estos trabajos no se prueba que la deficiencia de endorfinas sea el causante del STP $(14,15,16)$.

Efectos de los péptidos opiáceos*

\section{Disforia}

Labilidad emocional

Aumento del apetito y sed

Disminución hormona de crecimiento y prolactina.

Disminución actividad motoraDisminución inhibición tónica de gonadotropinas.

Aumento del interés sexual Aumento de glucagón e insulina.

Los péptidos opiáceos endógenos son considerados hoy por hoy por más de un autor, $(1,4,13,14,15)$ como agentes importantes en la fisiopatología del STP debido a que sus modificaciones durante el ciclo menstrual producen una serie de repercusiones bioquímicas que reunidas, son semejantes a varios de los principales síntomas del STP.

\section{Otras teorías:}

Existen muchísimas otras teorías sobre la etiología del STP; entre las cuales, mencionaremos la alergia endógena a las hormonas, propuesta por Reid (8), pero con numerosas incógnitas sin responder; también se escribió sobre disfunción piscosomática (17) concluyendo el mismo autor que existía muy poca evidencia para justificar esta hipótesis, hasta llegar a la hipótesis de las prostaglandinas propuesta por Budoff (18), ya que las mismas se encuentran en todo el organismo y es así como a nivel uterino son responsable de la dismenorrea funcional; en la hemorragia uterina disfuncional hay aumento en la producción de postaciclina por el endometrio; a nivel del sistema nervioso actúan como neurotransmisores y modifican la sed, apetito, estado de ánimo, llegando a actuar hasta en el riñón, intestinos y regulación del transporte de iones y líquidos; pero todo esto es considerado circunstancial y parece más bien que las prostanglandinas actúan como mediadores y que no son ellas las causantes del síndrome $(4,18)$.

De todas formas, a pesar de la información existente, todavía no se puede mencionar una teoría o hipótesis específica que explique el STP, pero estamos de acuerdo con Reid $(1,8)$ y con Strickler (4) en que las catecolaminas y los péptidos opiáceos endógenos son los neurotransmisores que más se ajustan al cuadro del STP, aunque ocasionalmente parezca que se alejan del mismo.

Con el objetivo de conocer la incidencia del STP en nuestro medio, -pues es desconocido-, decidimos hacer un estudio inicial al azar, entrevistando a grupos de estudiantes de carreras paramédicas entre 10. a 5o. año y de manera voluntaria. Se les repartió un cuestionario en el cual se consignaban los antecedentes ginecológicos, y la edad, si procedía de área rural o urbana; se investigaron 16 síntomas físicos, 9 psicológicos y 5 conductuales, también si existía algún factor que agravara los síntomas, si consultaban a su médico, y el número total de síntomas. Estos datos fueron luego tabulados y graficados para conocer los porcentajes y clasificar el STP en leve, moderado, y severo o intenso, de acuerdo al número de días de duración de los síntomas. También se investigó cuales drogas fueron más utilizadas.

\section{Resultados}

De el total de 317 entrevistas, se incluyeron 302 casos. Los rangos de edad fueron de 16 a 28 años con un promedio de 20.5 (Figura 1), estando el pico máximo entre los 19 y 21 años. El 56.62\% de los casos provenía del área urbana (Figura 2) y el $43.38 \%$ restante del área rural. La menarquía se presentó entre los 9 años $(=6)$ y 16 años $(n=4)$ con un promedio de 12.45 y una desviación estandard de 1.4 (Figura 3) estando el pico entre los 12 y 13 años. El inicio de los síntomas también tuvo rango entre los $9(\mathrm{n}=1)$ y 22 años $(\mathrm{n}=1)$ con un promedio de 14.86 y una desviación estandard de: 2.36 (Figura 4). El 55\% de las entrevistas utilizaban drogas para calmar o aminorar los síntomas (Figura 5), siendo la más utilizada el ácido mefenámico, seguida del

Figura 1

S.T.P. DISTRIBUCION POR EDAD

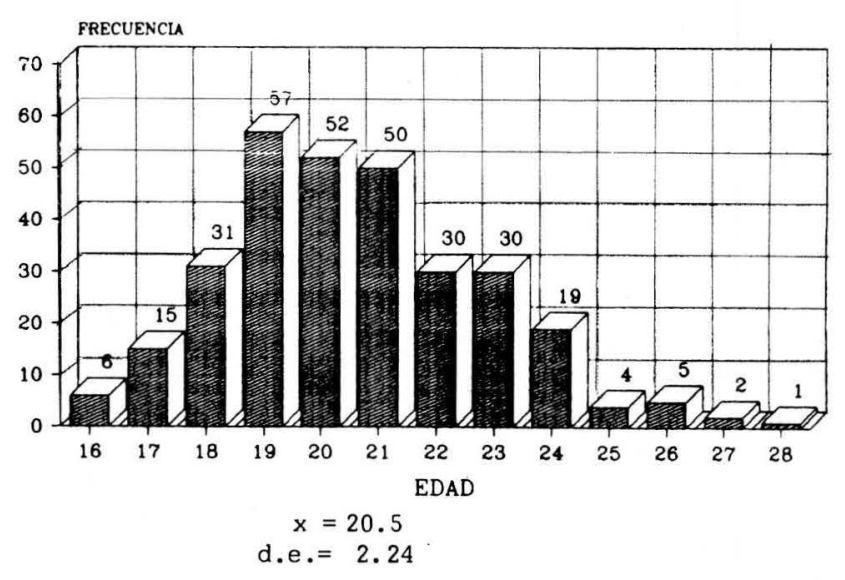

Figura 2

\section{S.T.P. DISTRIBUCION POR PROCEDENCIA}

\footnotetext{
* Tomado de Strickler. (4)
}

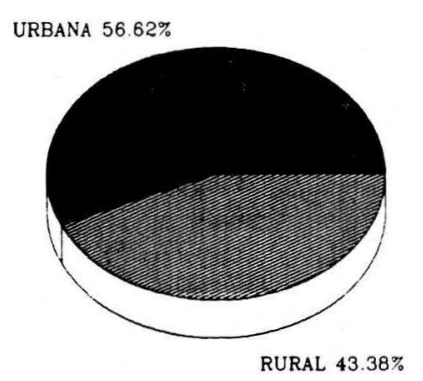


butilbromuro de hioscina, y nos llamó mucho la atención que menos del $1 \%$ consulta médicos o paramédicos y que básicamente es por autoformulación.

Tabla No. 1

SINDROME DE TENSION PREMENSTRUAL DISTRIBUCION POR EDADES

\begin{tabular}{lrr}
\hline Edades & Frecuencia & Porcentaje \\
\hline & & \\
16 & 6 & $1.96 \%$ \\
17 & 15 & $4.97 \%$ \\
18 & 31 & $10.26 \%$ \\
19 & 57 & $18.87 \%$ \\
20 & 52 & $17.22 \%$ \\
21 & 50 & $16.56 \%$ \\
22 & 30 & $9.93 \%$ \\
23 & 30 & $9.93 \%$ \\
24 & 19 & $\mathbf{6 . 6 2 \%}$ \\
25 & 4 & $\mathbf{1 . 3 2 \%}$ \\
26 & 5 & $\mathbf{1 . 6 6 \%}$ \\
27 & 2 & $\mathbf{0 . 6 6 \%}$ \\
28 & 1 & $0.30 \%$ \\
Total población & 302 & \\
X=20.5 & & \\
d.e.-2.24 & & \\
\hline
\end{tabular}

Figura 3

\section{S.T.P. INICIO MENARQUIA}

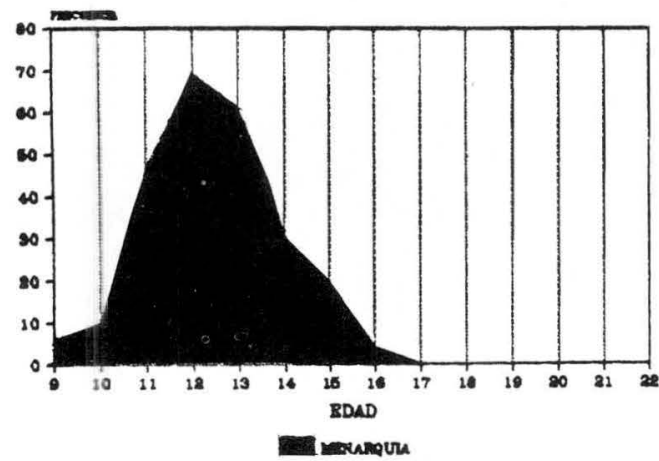

Figura 4

S.T.P. INICIO SINTOMAS

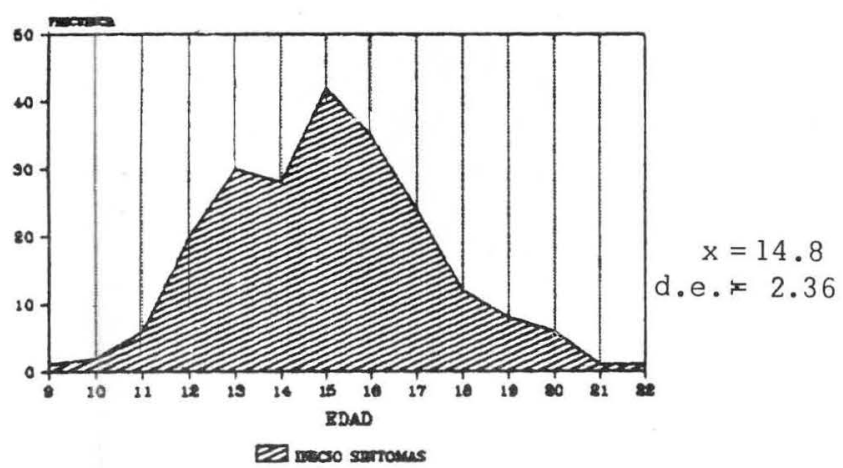

Figura 5

\section{S.T.P. DROGAS MAS UTILIZADAS}

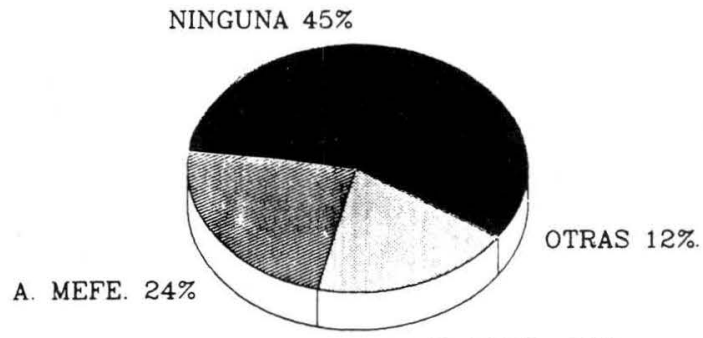

B. DE H. $19 \%$

Al analizar los síntomas físicos que más se presentan en nuestro medio encontramos (Figura 6) que el síntoma más común es la mastalgia, y se presentó en 207 casos (67.2\%), seguido por la hinchazón abdominal en 188 casos en 185 casos $(62.2 \%)$, el acné $(61.2 \%)$, cefalea en 150 casos (50\%), hinchazón de senos en 143 casos (47.35\%), el flujo vaginal en 109 casos $(36.09 \%)$ dolor articular y fatiga en 98 y 97 casos (32\%) y el resto de los síntomas por debajo del $25 \%$.

Entre los síntomas psicológicos tuvo predominio la irritabilidad con 176 casos (58.2\%), seguido por la depresión de 157 casos (52\%), la cólera se presentó en 100 entrevistas con $33.1 \%$, y sensibilidad al ruido en $28.8 \%$ (Figura 7 ).

Figura 6

\section{S.T.P. SINTOMAS FISICOS MAS COMUNES}

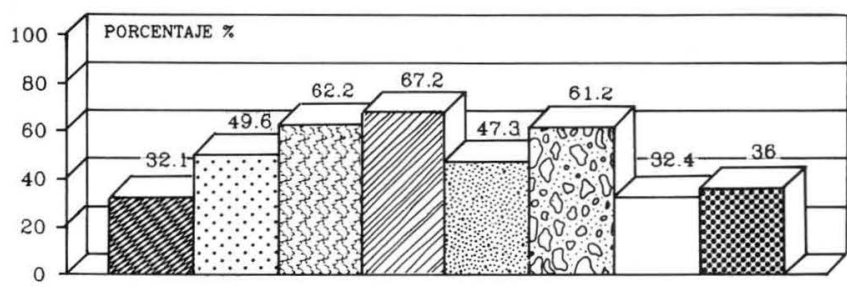

SINTOMA

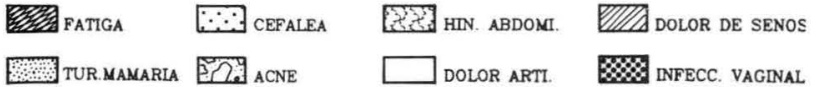

Figura 7

S.T.P. SINTOMAS PSICOLOGICOS

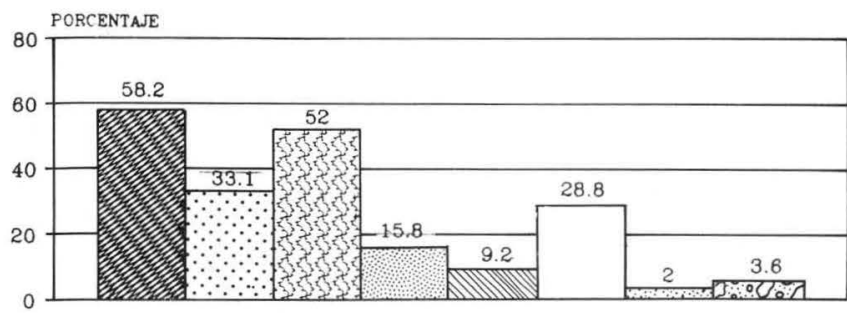

SINTOMA

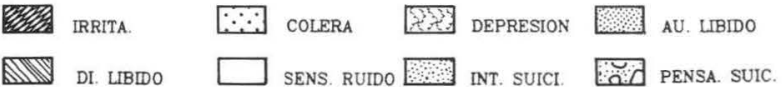


Entre los síntomas conductuales investigados (Figura 8), la afectación de la situación laboral y escolar fueron los más frecuentes en 16.8 y $15.2 \%$ respectivamente.

A el 54.6\% de las jóvenes éste STP no las afecta (Figura 9), pero el $17 \%$ se sienten incómodas, $9.6 \%$ se sienten inseguras.

Figura 8

\section{S.T.P. SINTOMAS CONDUCTUALES MAS COMUNES}

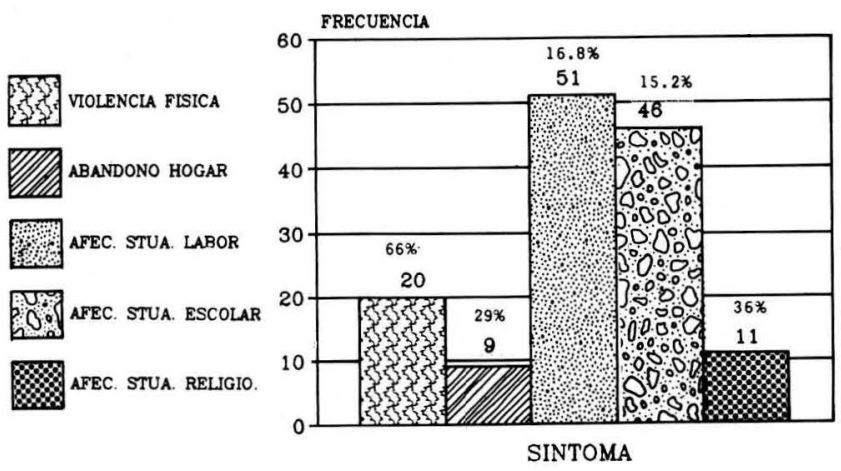

Figura 9

S.T.P. INFLUENCIA EN LA AUTOIMAGEN

PORCENTAJE

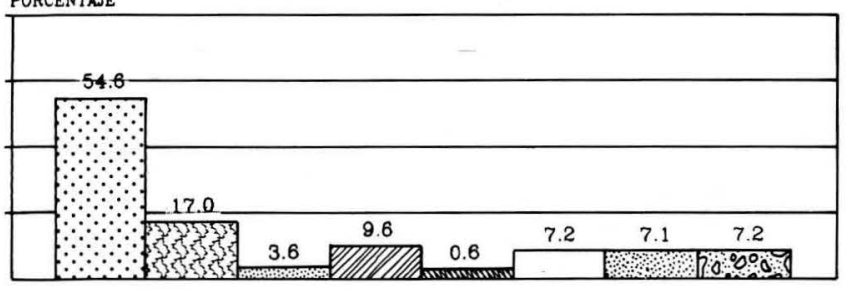

SINTOMA

$\because$ NO AFECTA $_{\text {SIENTE BONITA }} \square$ FACOMODIDAD
FASTIO

Figura 10

S.T.P. TOTAL DE SINTOMAS PRESENTADOS

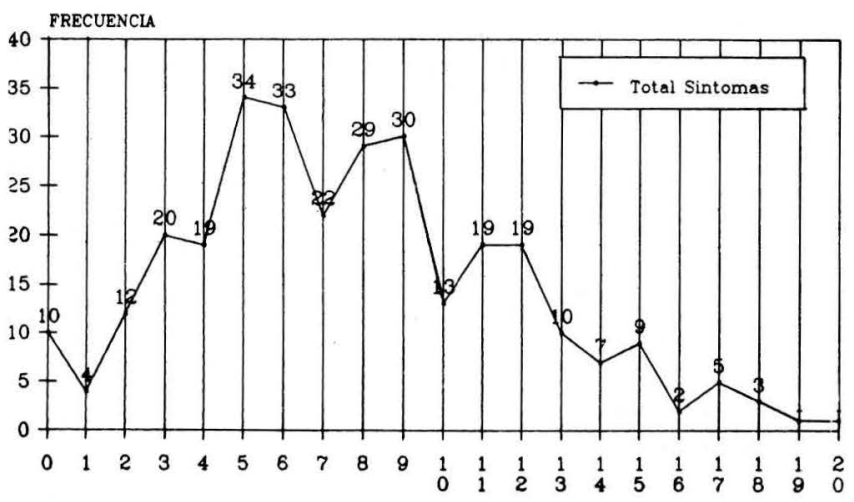

Al analizar el total de síntomas presentados por cada paciente (Figura 10) encontramos que 10 no tenían síntomas y 12 tenían entre 16 y 20 síntomas, estando el pico entre $5(11.25 \%)$ y $9(10 \%)$ síntomas.

En nuestro grupo entrevistado encontramos que en el $64.5 \%(n=194)$, los síntomas se presentaban entre horas y no más de 4 días antes del período menstrual y las clasificamos con STP leve, el $25.2 \%(n=76)$ los síntomas se iniciaban y duraban entre 4 a 8 días y las clasificamos como moderado y el $73 \%(n=22)$ los síntomas tenían duración de más de 8 días y las clasificamos como severo o intenso (figuras: 11,12,13). Sólo el 3.2\% $(n=10)$ de las entrevistadas no tuvieron ningún síntoma.

Figura 11

\section{S.T.P. DIAS DE INICIACION DE SINTOMAS}

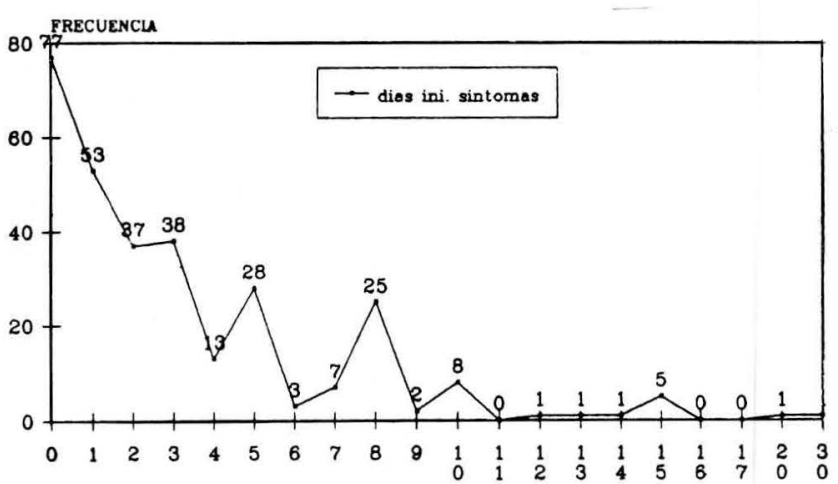

Figura 12

S.T.P. DIAS DE DURACION DE SINTOMAS

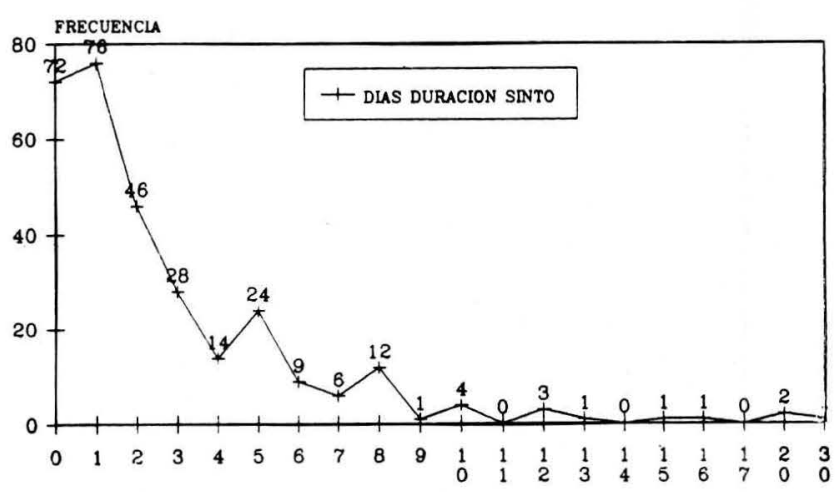

Figura 13

S.T.P. DISTRIBUCION POR NIVELES

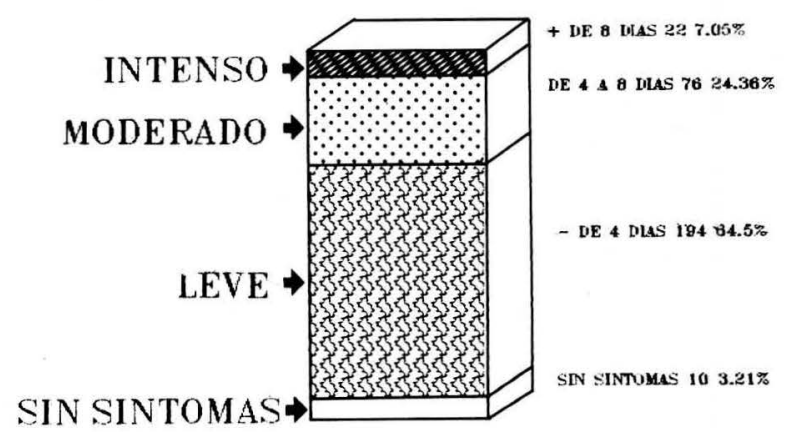




\section{Comentarios}

Como es bien sabido, los síntomas deI STP son numerosísimos y algunos autores $(2,19)$ llegan a encontrar hasta más de 100 síntomas; otros como Moos (20) han elaborado cuestionarios con 47 síntomas, divididos en 8 grandes grupos y Magos (21) tienen un cuestionario con 34 síntomas escogidos entre los más frecuentes reportados por Moos (20); nosotros utilizanos un cuestionario con 30 síntomas, dividido en tres grupos (Figura 6,7,8) pues nos pareció mejor por ser un estudio inicial. Analizando la edad, encontramos que el promedio nuestro es mucho más bajo que los publicados por Keyer (22) y Maghos (21) pues estos tienen 34 años en promedio con rango de 14 a 55 y 34.3 años con rangos de 22 a 49 respectivamente; y consideramos esto debido a que nuestro grupo de pacientes con un promedio de 20.5, estuvo conformado por jóvenes universitarias entre los 16 y 28 años que además de ser más jóvenes, es un grupo más homógeneo: Observamos también que independientemente de la edad, las mujeres de nuestro grupo no acostumbran a visitar médicos y que gran número se automedican (Figura 5), a diferencia en otros países donde por el control que existe con los medicamentos, además que no consideran tan natural los síntomas de STP, éstas tienen, según lo publicado (22) un promedio de hasta de tres médicos por paciente, psicólogos, trabajadores sociales y profesionales afines y según otros (21) hasta $86 \%$ de las mujeres habían recibido tratamiento y consulta, y nosotros en nuestro medio no alcanzamos ni siquiera el $1 \%$ de consulta médica y el $55 \%$ se automedican.

También encontramos variación en la fecha de inicio de los síntomas, pues Keyer (22) tiene que el $52 \%$ se produce después de un parto y sólo el $35 \%$ aparece espontáneamente en la adolescencia y en nuestro grupo en casi todas se inicia desde la adolescencia, aproximadamente 2.4 años posterior a la menarquia, no encontrándose factor desencadenante o que agraven los síntomas en $63.6 \%$ y entre el $36.4 \%$ restante están el estress, el frío y el ejercicio entre otros; (Tabla No. 1) no encontramos ningún cáso en que el factor desencadenante haya sido un aborto o infección genital, como ya ha sido considerado (2).

El promedio de días de inicio de los síntomas obtenidos fue de 7.8 y la duración de 5.20 , lo cual está entre los promedios publicados con anterioridad por algunos autores $(1,2,3,13)$ aunque un poco menos que otros $(21,22)$.

En lo referente a los síntomas físicos (Figura 6) nuestros resultados están de acuerdo con lo publicado $(1,3,8,19$, $20,21,22)$ ya que la mastalgia, la hinchazón abdominal, el acné, la cefalea, el edema mamario, el flujo vaginal, la fatiga y el dolor articular, están entre los más comunes, variando solamente los porcentajes y el orden; así, Keyer tiene en primer lugar la fatiga con $98 \%$ mientras que nosotros la tenemos en octavo con $32.1 \%$; las artralgias las tiene en sexto puesto con $73 \%$ y nosotros en séptimo con $32.4 \%$ y consideramos esto es debido a la diferencia cultural y de latitudes de ambos países.
Entre los síntomas psicológicos, la irritabilidad y la depresión fueron los más comunes (Figura 7) que también coordina con los autores ya mencionados; pero al compararlos con el trabajo de Keyer, que incluye porcentajes, también nos llama la atención que, como en los síntomas físicos, sus porcentajes son mucho más elevados que los nuestros (97-98\% v.s. $58.51 \%)$. pero conserva como más comunes la irritabilidad, la depresión y la ansiedad.

En lo que se refiere a los intentos o pensamientos suicidas, tuvimos sólo $2 \%$ y $3.6 \%$ respectivamente y aunque se encuentran estos síntomas hasta $20 \%(22)$, es muy importante realizar la diferenciación entre estas dos entidades (23), pues se ha encontrado que existe en pacientes con STP algún tipo de afecciones psiquiátricas y por lo tanto aconsejan estos autores (23) que se grafiquen los síntomas en estos pacientes y si no existe un aumento definido de los síntomas psicóticos en la fase lutea, la paciente puede tener una alteración psiquiátrica subyacente y debe tratársele.

Los síntomas conductuales fueron los que se presentaron con menos frecuencia y sus resultados están claros (Figura 8). Al analizar el total de síntomas encontramos que el promedio obtenido por nosotros de síntomas (Figura 10) coordina con lo publicado $(20,21,22)$.

Al realizar la clasificación final de nuestro grupo de pacientes encontramos que también están de acuerdo con lo publicado anteriormente $(1,2,13,21)$ teniendo aproximadamente $64.5 \%$ de pacientes con STP leve, $25.2 \%$ con STP moderado y $7.3 \%$ pacientes con STP severo o intenso, y de el total de las 302 mujeres sólo $3.2 \%$ no presentaron ningún síntoma (Figura 13).

\section{Conclusiones}

1. El 64.5\% de nuestras pacientes cursan con STP leve, el $25.2 \%$ con STP moderado y el $7.3 \%$ con STP severo.

2. Menos de $1 \%$ de las mujeres de nuestro estudio consultan por alguno de los síntomas presentados, y el $55 \%$ de ellas se automédica.

3. El promedio de edad fue de 20.5 años y el $56.3 \%$ proviene de área urbana.

4. Los síntomas físicos más comunes fueron mastalgia $(67.2 \%)$, hinchazón abdominal (66.2\%), acné $(61 \%)$ y cefalea (50\%).

5. Los síntomas psicológicos predominantes fueron la irritabilidad (58.3\%) y la depresión (52\%) y de los síntomas conductuales fueron la afectación laboral y escolar.

6. No existió correlación entre la edad, lugar de procedencia ni menarquia, con la intensidad y duración de los síntomas, ni con la clase de síntomas (físicos, psicológicos y conductuales).

\section{Agradecimientos}

A la señora Leomy Durán por la trascripción del manuscrito, al Psicólogo Hugo Rodríguez por la elaboración de gráficas y cálculos y al Laboratorio Schering que patrocinó la presentación del trabajo. 


\section{BIBLIOGRAFIA}

1. Reid, Robert; Yen S.S.C: El Síndrome Premenstrual. Clin Obst Ginecol. Editorial Interamericana. México D.F. 1983; Vol. 3; 863-874.

2. Praxis Médica Ediciones Latinoamericanas S.A. España, 10:160-165.

3. Jones, Howard Jones, G.S.: Tratado de Ginecología de Novak. Editorial Interamericana. México 10a. Edición 1984; 836-839.

4. Strickler, Ronald:; Hipótesis de la etiología endocrina del síndrome premenstrual. Clin. Obstet. Ginecol. Editorial Interamericana. México D.F., 1987; Vol. 2: 359-366.

5. Frank, R.T. The hormonal basis of premenstrual tensión. Arch Neurol. Psychiatr., 1931; 26: 1053-57 (Citado por Strickler(4))

6. Backstrom, T. Sanders. D. Leask, R: et al.: Mood sexuality, hormonas, an the menstrual cicle. II. Hormones Leveis and their relationship to the premenstrual syndrome. Psychosom Med, 1983; 45: 503-7.

7. Dalton. K. Cyclical criminal acts in premenstrual syndrome. Lancet, 1980; 2:1070.

8. Reid, Robert; Yen, S.S.C. Premenstrual syndrome. Am J Obstet Gynecol, 1981; 139: 85-104.

9. Parker C.R. Winkel, Ca. et al: Plasma concentrations of 11- desoxycorticosterone in women during the menstrual cycle Obstet Ginecol 1981; 58: 26-30.

10. Andersch, B.; Bromocriptine and premenstrual symptomes; a survey of double blind trials. Obstet Ginecol. Surv 1983; 38: 643-6.

11. Reid, R.L.; Greenway-Caotes, A.: Hahn, P; Oral glucose tolerance during the menstrual cycle in normal and women with alleged premenstrual "hypoglycemic" attackes effects of naloxone. J Clin Endocrinol Metabol. 1986; 62: 1167-72.

12. Adams, P. Rose D. et al: Effect of pyridoxine hydrochloride (vitamina B6) upon depression. Lancet. 1973; 1: 897-904.
13. González Merlo, J.; Ginecología. Editorial Salvat. España 5a. Edición 1988; 193-195.

Qtingley, M; Yen, S.S.C. The role of endoge nous opiates on LH secretion during the menstrual cycle J Clin Endocrino Metab 1980; 51: 179 .

15. Chuong, C.J; Coulam, C.B. et al: Neuropeptide levels in premenstrual syndrome. Fertil Steril. 1985; 44: 760-765.

16. Peck, S.D. Can increased beta-endorphins explain the etiology of premenstrual syndrome J Am Osteopath Assc. 1982; 82: 192-7

17. Ganon L. Evidence for a psychological etiology of menstrual disorders a critical review. Psycholgical Reports. 1981; 17: 287. (Citado por Reid 1).

18. Budoff, Penny W. Uso de inhibidores de las prostaglandinas en el Tratamiento de Síndrome Premenstrual. Clínica Obst Ginecol Editorial Interamericana. México D.F., 1987; Vol. 2: 429-439.

19. Smith, Samuel; Schiff, Isaac.: The premenstrual syndrome diagnosis an management. Fertil Steril. 1989; Vol. 52(4): 527-543.

20. Moos, R.H.: Typology of menstrual cycle symptome. Am J Obstet Gynecol 1969; 103: 390-4

21. Magos, A.L.; Brincat, M. and Stud, J.W.: Trend analyzis of 150 women with a history of the premenestrual syndrome. Am J Obstet Gynecol 1986; 155(2): 277-82.

22. Keyer, W: Valoración general de los síntomas premenstruales. Clin Obstet Ginecol 1983; Vol. 26(3): 377-386.

23. Stout. A; Steege, J.: Blazer, D. and George, L. Comparación de los diagnósticos psiquiátricos permanentes en consultorios del síndrome premenstrual y muestras comunitarias. J Nerv Ment Dise 1986; 174: 517-22.

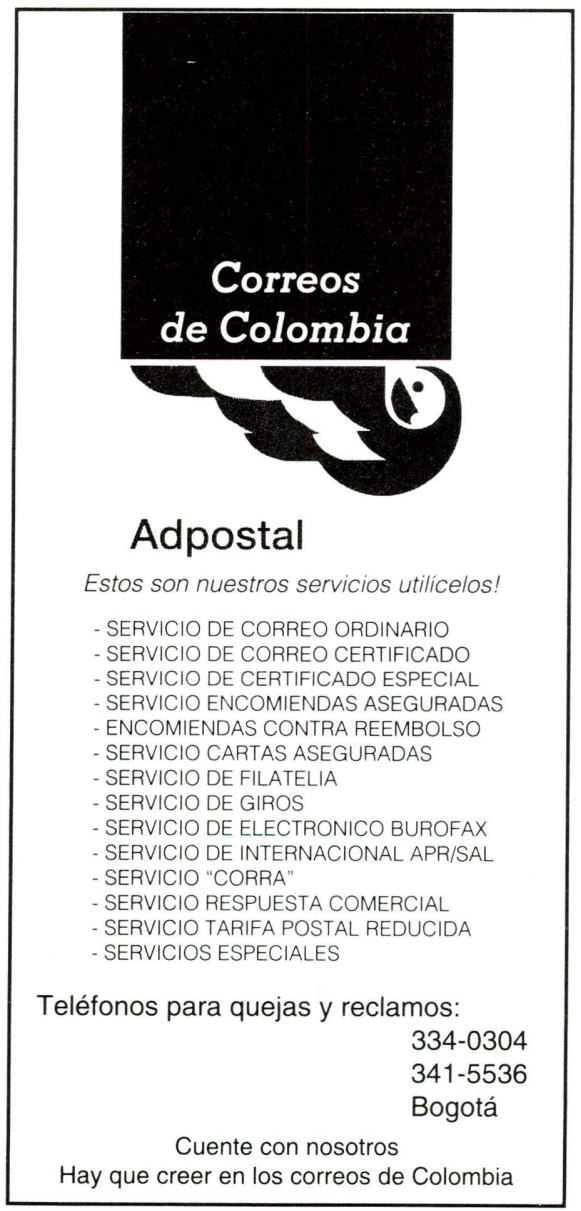

